\title{
LOW DIMENSIONAL HOMOTOPY FOR MONOIDS II: GROUPS
}

\author{
STEPHEN J. PRIDE \\ Department of Mathematics, University of Glasgow, University Gardens, Glasgow G12 8QW, Scotland
}

(Received 28 January, 1997)

Introduction. Consider a group presentation

$$
\hat{\mathcal{P}}=<\mathbf{x} ; \boldsymbol{r}>\text {. }
$$

Here $\mathbf{x}$ is a set and $\mathbf{r}$ is a set of non-empty, cyclically reduced words on the alphabet $\mathbf{x} \cup \mathbf{x}^{-1}$ (where $\mathbf{x}^{-1}$ is a set in one-to-one correspondence $x \leftrightarrow x^{-1}$ with $\mathbf{x}$ ). We assume throughout that $\hat{\mathcal{P}}$ is finite. Let $\hat{F}$ be the free group on $\mathbf{x}$ (thus $\hat{F}$ consists of free equivalence classes [ $W$ ] of word on $\mathbf{x} \cup \mathbf{x}^{-1}$ ), and let $N$ be the normal closure of $\{[R]$ : $R \in \mathbf{r}\}$ in $\hat{F}$. Then the group $G=G(\hat{\mathcal{P}})$ defined by $\hat{\mathcal{P}}$ is $\hat{F} / N$. We will write $W_{1}={ }_{G} W_{2}$ if $\left[W_{1}\right] N=\left[W_{2}\right] N$.

Associated with $\hat{\mathcal{P}}$ is a certain crossed module $(\Sigma, \hat{F}, \partial)$. This can be described in several different (but equivalent) ways:

(a) topologically as the relative second homotopy group $\pi_{2}\left(\mathcal{K}, \mathcal{K}^{(1)}\right)$ where $\mathcal{K}$ is the standard 2-complex modelled on $\hat{\mathcal{P}}$ and $\mathcal{K}^{(1)}$ is its 1 -skeleton;

(b) algebraically in terms of sequences;

(c) geometrically in terms of pictures.

Also, there is the (absolute) second homotopy group $\pi_{2}(\hat{\mathcal{P}})=\operatorname{Ker} \partial$, which is a $Z G$ module. Elements of this can be represented algebraically by identity sequences, or geometrically by spherical pictures. See [1], [3], [10] for details. We will use the second description (b), and refer the reader to [10] for basic terminology and results concerning identity sequences. (However, for the reader's convenience we give a brief account of this material in $\S 1$ below.)

Now $\hat{\mathcal{P}}$ gives rise to a monoid presentation $\mathcal{P}$ for $G$, where

$$
\mathcal{P}=\left[\mathbf{x}, \mathbf{x}^{-1} ; R=1(R \in \boldsymbol{r}), x^{\varepsilon} x^{-\varepsilon}=1(x \in \mathbf{x}, \varepsilon= \pm 1)\right] .
$$

The monoid defined by $\mathcal{P}$ is the quotient of the free monoid $F$ on $\mathbf{x} \cup \mathbf{x}^{-1}$ by the smallest congruence $\rho$ generated by the relations. A typical element of this monoid is a congruence class $W \rho(W \in F)$, and we have an isomorphism from this monoid to $G$, given by

$$
W \rho \mapsto[W] N(W \in F) .
$$

We will often identify $W \rho$ and $[W] N$ (if no confusion can arise) and will denote this element by $\bar{W}$.

Now in [12] (see also [11]) we associated with any monoid presentation $\mathcal{Q}$ a 2 complex $\mathcal{D}(\mathcal{Q})$ ("the 2-complex of monoid pictures") and we showed that the first homology group $H_{1}(\mathcal{D}(\mathcal{Q})$ ) has considerable significance. The fundamental groups of $\mathcal{D}(\mathcal{Q})$ are also of considerable interest and have been investigated by Guba and Sapir [7], and Kilibarda [8].

For our presentation $\mathcal{P}$ above, the 2-complex $\mathcal{D}(\mathcal{P})$ has underlying graph as follows. The vertex set is $F$ and the edge set consists of all the atomic monoid 
pictures $(U, T, \varepsilon, V)\left(U, V \in F, T \in \mathbf{r} \cup\left\{x x^{-1}, x^{-1} x: x \in \mathbf{x}\right\}, \varepsilon= \pm 1\right)$ (Figure 1). The initial, terminal and inversion functions $\iota, \tau,{ }^{-1}$ are given by

$$
\begin{aligned}
\iota(U, T, 1, V) & =\tau(U, T,-1, V)=U T V, \\
\iota(U, T,-1, V) & =\tau(U, T, 1, V)=U V, \\
(U, T, \varepsilon, V)^{-1} & =(U, T,-\varepsilon, V) .
\end{aligned}
$$

There are obvious (compatible) left and right actions of $F$ on this graph. Paths in this graph are called (monoid) pictures. The left and right actions of $F$ extend to actions on pictures. The defining paths of $\mathcal{D}(\mathcal{P})$ are the paths

$$
[\mathrm{A}, \mathrm{B}]=(\mathrm{A} \cdot \iota(\mathrm{B}))(\tau(\mathrm{A}) \cdot \mathrm{B})\left(\mathrm{A}^{-1} \cdot \tau(\mathrm{B})\right)\left(\iota(\mathrm{A}) \cdot \mathrm{B}^{-1}\right) .
$$

(A,B are edges of the graph.) See [11], [12] for further details.

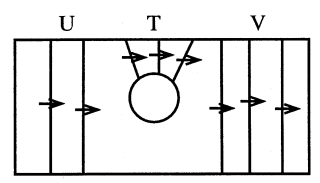

$(\mathrm{U}, \mathrm{T},+1, \mathrm{~V})$

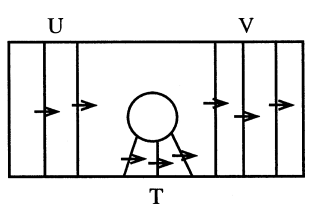

(U, T, -1, V)

Figure 1

Now elements of the fundamental groupoid $\pi_{1}(\mathcal{D}(\mathcal{P}))$ are represented by monoid pictures. Consequently, in view of (c) above, it is natural to ask for our group $G$ what is the relationship (if any) between $\pi_{1}(\mathcal{D}(\mathcal{P})$ ) and $\Sigma$.

In fact to obtain a relationship we need to modify $\mathcal{D}(\mathcal{P})$ by adding some extra defining paths to it. For each $x \in \mathbf{x}, \varepsilon \pm 1$ we have the spherical monoid picture as in Figure 2. (This is a path of length 2 in $\mathcal{D}(\mathcal{P})$.) We let $\mathcal{D}(\mathcal{P})^{*}$ be the 2-complex obtained from $\mathcal{D}(\mathcal{P})$ by adding the extra defining paths

$$
W \cdot \mathrm{P} \cdot V \quad(P \text { as in Figure } 2, W, V \in F) .
$$

Now let $\Sigma^{*}$ be the collection of all elements of the fundamental groupoid $\pi_{1}\left(\mathcal{D}(\mathcal{P})^{*}\right)$ represented by monoid pictures which start at freely reduced words on $\mathbf{x}$ $\cup \mathbf{x}^{-1}$, and end at the empty word. We show in $\S 2$ that a crossed module structure $\left(\Sigma^{*}, \hat{F}, \partial^{*}\right)$ can be imposed on $\Sigma^{*}$, and we prove (Theorem 1$)$ that there is a crossed module isomorphism

$$
\psi: \Sigma \rightarrow \Sigma^{*}
$$

By restriction, we then get a $Z G$-isomorphism

$$
\pi_{2}(\hat{\mathcal{P}})=\operatorname{Ker} \partial \stackrel{\Psi}{\rightarrow} \operatorname{Ker} \partial^{*}=\pi_{1}\left(\mathcal{D}(\mathcal{P})^{*}, 1\right) .
$$

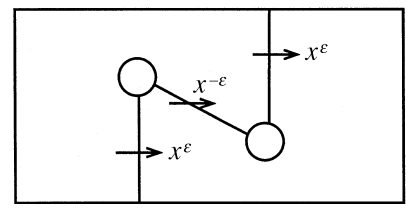

Figure 2 
The notion of finite derivation type (FDT) was introduced by Squier in his posthumously published article [13]. In our terminology, a monoid presentation $\mathcal{Q}$ is $F D T$ if there is a finite set $X$ of spherical monoid pictures over $\mathcal{Q}$ such that the 2complex $\mathcal{D}(\mathcal{Q})^{X}$ obtained from $\mathcal{D}(\mathcal{Q})$ by adding the defining paths

$$
W \cdot \mathrm{P} \cdot V \quad(W, V \in F, \mathrm{P} \in X)
$$

has trivial fundamental groups. A finitely presented monoid $S$ is $F D T$ if some (and hence, as shown by Squier [13], any) finite presentation of $S$ is $F D T$. Monoids of finite derivation type have been discussed in [4], [5], [9], [12].

Now if $G$ is a group then it has been shown by Cremanns and Otto [5] that $G$ is $F D T$ if and only if for some (and hence, in fact, any) finite group presentation $\hat{\mathcal{P}}$ of $G$, the $\mathrm{Z} G$-module $\pi_{2}(\hat{\mathcal{P}})$ is finitely generated.

We give in $\$ 3$ a simple proof of the Cremanns/Otto result mentioned above. Let $\hat{\mathcal{P}}, \mathcal{P}$ be as in (1), (2) respectively. We first establish the easy fact that all the fundamental groups of $\mathcal{D}(\mathcal{P})^{*}$ are isomorphic. Using this we prove (Theorem 2) that $\mathcal{P}$ is $F D T$ if and only if the $\mathrm{Z} G$-module $\pi_{1}\left(\mathcal{D}(\mathcal{P})^{*}, 1\right)$ is finitely generated. Then in view of the isomorphism $\pi_{2}(\hat{\mathcal{P}}) \cong \pi_{1}\left(\mathcal{D}(\mathcal{P})^{*}, 1\right)(\S 2)$, the Cremanns/Otto result follows.

It should be noted that for any group presentation $\hat{\mathcal{P}}=\langle\mathbf{x} ; \mathbf{r}\rangle$ there is a standard exact sequence

$$
0 \rightarrow \pi_{2}(\hat{\mathcal{P}}) \rightarrow \oplus_{R \in \mathbf{r}} Z G e_{R} \rightarrow \oplus_{x \in \mathbf{x}} Z G e_{x} \rightarrow Z G \rightarrow Z \rightarrow 0
$$

of $\mathrm{ZG}$-modules (see for example [3], [10]). Using this, together with the generalised Schanuel Lemma [2], one easily obtains the (well-known) result that a finitely presented group $G$ is of type $\mathrm{FP}_{3}[2]$ if and only if for some (in fact any) finite presentation $\hat{\mathcal{P}}$ of $G, \pi_{2}(\hat{\mathcal{P}})$ is finitely generated. Thus, for finitely presented groups, $F D T$ and $\mathrm{FP}_{3}$ are equivalent. (This result is obtained in [5].)

1. Preliminaries. If $\mathrm{P}, \mathrm{P}^{\prime}$ are paths in $\mathcal{D}(\mathcal{P})^{*}$ then we write $\mathrm{P} \sim \mathrm{P}^{\prime}$ if $\mathrm{P}, \mathrm{P}^{\prime}$ are equivalent (homotopic) in $\mathcal{D}(\mathcal{P})^{*}$. The equivalence class of $\mathrm{P}$ will be denoted by $<\mathrm{P}\rangle$. We will assume the reader has some familiarity with the material regarding monoid pictures in $[\mathbf{1 2}, \S \S 2,5]$.

An edge of $\mathcal{D}(\mathcal{P})^{*}$ of the form $\left(U, x^{\varepsilon} x^{-\varepsilon}, \pm 1, V\right)(U, V \in F, x \in \mathbf{x}, \varepsilon= \pm 1)$ will be called trivial, and a path will be called trivial if all its edges are trivial. Two vertices $W_{1}, W_{2}$ can be connected by a trivial path if and only if $W_{1}$ and $W_{2}$ are freely equivalent (the chosen path connecting $W_{1}$ to $W_{2}$ then gives a method of freely transforming $W_{1}$ to $W_{2}$ ). In view of the defining paths $(3)$ of $\mathcal{D}(\mathcal{P})^{*}$, we have that any two trivial paths between a given pair of vertices $W_{1}, W_{2}$ are homotopic in $\mathcal{D}(\mathcal{P})^{*}$. This key observation allows us to replace a trivial subpath $\mathrm{T}$ of a given path $\mathrm{P}$ by any other trivial path $\mathrm{T}^{\prime}$ (where $\iota\left(\mathrm{T}^{\prime}\right)=\iota(\mathrm{T}), \tau\left(\mathrm{T}^{\prime}\right)=\tau(\mathrm{T})$ ) without affecting the homotopy type of $\mathrm{P}$.

Suppose $\mathrm{P}$ is a path in $\mathcal{D}(\mathcal{P})^{*}$ with $\iota(\mathcal{P})=W, \tau(\mathrm{P})=Z$, and let $\mathrm{T}, \bar{T}$ be trivial paths in $\mathcal{D}(\mathcal{P})^{*}$ from $W_{1}$ to $W, Z_{1}$ to $Z$ respectively, where $W_{1}, Z_{1}$ are the unique reduced words freely equivalent to $W, Z$. Then the picture $T P \bar{T}^{-1}$ will be said to be obtained from $\mathrm{P}$ by freely reducing the boundary of $\mathrm{P}$, and will be denoted by $\mathrm{P}^{*}$. Obviously this notation is ambiguous because $\mathrm{P}^{*}$ depends on $\mathrm{T}, \bar{T}$. However, since we will be working up to homotopy in $\mathcal{D}(\mathcal{P})^{*}$, we can, by our comment in the previous paragraph, allow ourselves to choose any trivial paths $\mathrm{T}, \bar{T}$ that suit our purpose. This simple, but key point will be used over and over again, without further comment. 
Another important point is the following.

Suppose that $P_{1}$ is obtained from $P$ by inserting into $P$ a pair of parallel arcs with labels $x^{\varepsilon}, x^{-\varepsilon}(x \in \mathbf{x}, \varepsilon= \pm 1)$. Then $P_{1}^{*} \sim P^{*}$.

This is because, when we freely reduce the boundary of $\mathrm{P}_{1}$ we can begin as in Figure 3. This creates a cancelling pair of discs which can be removed.

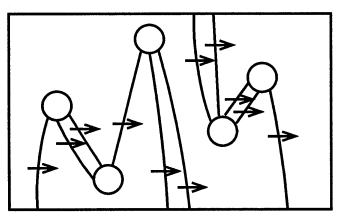

$\mathbb{P}$

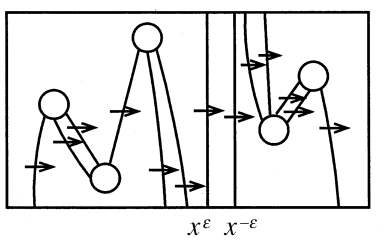

$\mathbb{P}_{1}$

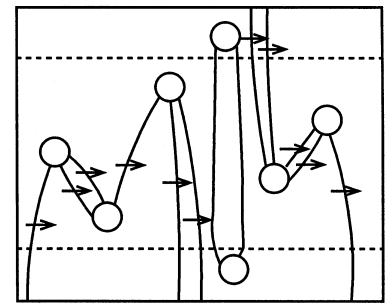

Figure 3

If $\mathrm{P}, \mathrm{P}^{\prime}$ are paths in $\mathcal{D}(\mathcal{P})^{*}$ then we write $\mathrm{P}+\mathrm{P}^{\prime}$ for the path $\left(\mathrm{P} \cdot \iota\left(\mathrm{P}^{\prime}\right)\right)\left(\tau(\mathrm{P}) \cdot \mathrm{P}^{\prime}\right)$. Then for paths $\mathrm{P}_{1}, \mathrm{P}_{2}, \cdots, \mathrm{P}_{n}$ we define $\mathrm{P}_{1}+\mathrm{P}_{2}+\ldots+\mathrm{P}_{n}$ inductively to be $\left(\mathrm{P}_{1}\right.$ $\left.+\ldots \mathrm{P}_{n-1}\right)+\mathrm{P}_{n}$.

For any $U \in F$, say $U=x_{1} x_{2} \cdots x_{m}\left(x_{i} \in \mathbf{x} \cup \mathbf{x}^{-1}\right.$ for $\left.i=1, \cdots, m\right)$ we denote the picture

$$
\prod_{i=1}^{m}\left(x_{1} \cdots x_{i-1}, x_{i} x_{i}^{-1},-1, x_{i-1}^{-1} \cdots x_{1}^{-1}\right)
$$

(see Figure 4) by $T_{U U^{-1}}$.

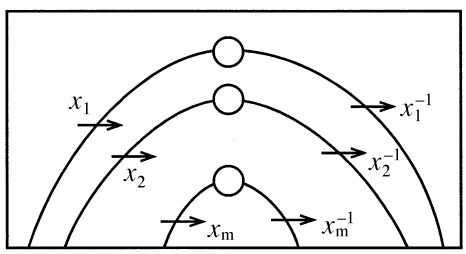

Figure 4

For $R \in \mathbf{r}, U \in F, \varepsilon \in\{-1,1\}$ we define $\mathrm{E}_{R, U, \epsilon}$ as follows:

$$
E_{R, U, \varepsilon}=\left\{\begin{array}{cc}
\left(U, R, 1, U^{-1}\right) & \varepsilon=1 \\
\left(U, R,-1, R^{-1} U^{-1}\right) & \varepsilon=-1
\end{array}\right.
$$

We complete this section by giving a brief account of $\Sigma$ in terms of sequences. (For further details, as well as for the elementary theory of crossed modules, see [10]. See also [6] for the theory of crossed modules.)

Let $\mathbf{r}^{F}$ be the set of all elements of $F$ of the form $W R^{\varepsilon} W^{-1}(W \in F, R \in \mathbf{r}, \varepsilon= \pm 1)$. We consider finite sequences $\sigma=\left(c_{1}, c_{2}, \cdots, c_{m}\right)$ of elements of $\mathbf{r}^{F}$. We define certain operations on sequences as follows.

(I) Replace some term $c_{i}=W R^{\varepsilon} W^{-1}$ by $c_{i}^{\prime}=W^{\prime} R^{\varepsilon} W^{\prime-1}$ where $W^{\prime}$ is a word freely equivalent to $W$. 
(II) Delete two consecutive terms if one is identically equal to the inverse of the other.

(III) Replace two consecutive terms $c_{i}, c_{i+1}$ by $c_{i+1}, c_{i+1}^{-1} c_{i} c_{i+1}$ or by $c_{i} c_{i+1} c_{i}^{-1}, c_{i}$.

Two sequences $\sigma, \sigma^{\prime}$ are said to be (Peiffer) equivalent if one can be obtained from the other by a finite number of operations (I), (II), (II $)^{-1}$, (III). The equivalence class containing $\sigma$ is denoted by $\langle\sigma\rangle$. The set $\Sigma$ of equivalence classes forms a (non-abelian) group under the binary operation

$$
<\sigma_{1}>+<\sigma_{2}>=<\sigma_{1} \sigma_{2}>\text {. }
$$

There is a (well-defined) action of $\hat{F}$ on $\Sigma$ given by

$$
[W] \cdot\langle\sigma\rangle=<\sigma^{W}>
$$

(where, if $\sigma=\left(c_{1}, \cdots, c_{m}\right)$ then $\sigma^{W}=\left(W c_{1} W^{-1}, \ldots, W c_{m} W^{-1}\right)$ ), and there is a group homomorphism

$$
\partial: \Sigma \rightarrow \hat{F}, \quad<\left(c_{1}, c_{2}, \cdots, c_{m}\right)>\mapsto\left[c_{1} c_{2} \cdots c_{m}\right] .
$$

The triple $(\Sigma, \hat{F}, \partial)$ then has the structure of a crossed module. A well-known result (originally proved by Whitehead [14]) is that this crossed module is free, with basis consisting of the elements $b_{R}=\langle(R)>(R \in \mathbf{r})$. By the elementary theory of crossed

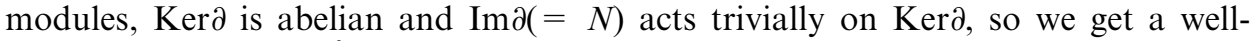

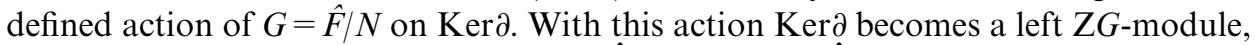
which is the second homotopy module of $\hat{\mathcal{P}}$, denoted $\pi_{2}(\hat{\mathcal{P}})$.

2. The crossed module $\Sigma^{*}$. We define a crossed module $\left(\Sigma^{*}, \hat{F}, \partial^{*}\right)$ as follows. The elements of $\Sigma^{*}$ are the equivalence classes $<\mathrm{P}>$ where $\mathrm{P}$ is a monoid picture such that $\iota(\mathrm{P})$ is a freely reduced word on $\mathbf{x} \cup \mathbf{x}^{-1}$ and $\tau(\mathcal{P})$ is the empty word. We define a (non-commutative) operation + on $\Sigma^{*}$ by

$$
<P_{1}>+<P_{2}>=<\left(P_{1}+P_{2}\right)^{*}>\left(<P_{1}>,<P_{2}>\in \Sigma^{*}\right),
$$

and an action (which is well-defined by (4)) of $\hat{F}$ on $\Sigma^{*}$ by

$$
[W] \circ<P>=<\left(W \cdot P \cdot W^{-1}\right)^{*}>\left([W] \in \hat{F},<P>\in \Sigma^{*}\right) .
$$

We define

$$
\partial^{*}: \Sigma^{*} \rightarrow \hat{F}
$$

by

$$
\partial^{*}<P>=[\iota(P)]\left(<P>\in \Sigma^{*}\right) .
$$

Then under the operation,$+ \Sigma^{*}$ is a group on which $\hat{F}$ acts. Clearly, for $[W] \in \hat{F}$, $<\mathrm{P}>\in \Sigma^{*}$ we have

$$
\partial^{*}([W] \circ<P>)=[W] \partial^{*}<P>[W]^{-1} .
$$


Also, as can be seen geometrically (Figure 5), for any $<\mathrm{P}_{1}>,<\mathrm{P}_{2}>\in \Sigma^{*}$ we have

$$
<P_{1}>+<P_{2}>=\partial^{*}<P_{1}>0<P_{2}>+<P_{1}>\text {. }
$$

Thus $\left(\Sigma^{*}, \hat{F}, \partial^{*}\right)$ is a crossed module. Note that

$$
-<P>=<\left(P^{-1} \cdot \iota(P)^{-1}\right)^{*}>\quad\left(<P>\in \Sigma^{*}\right) .
$$

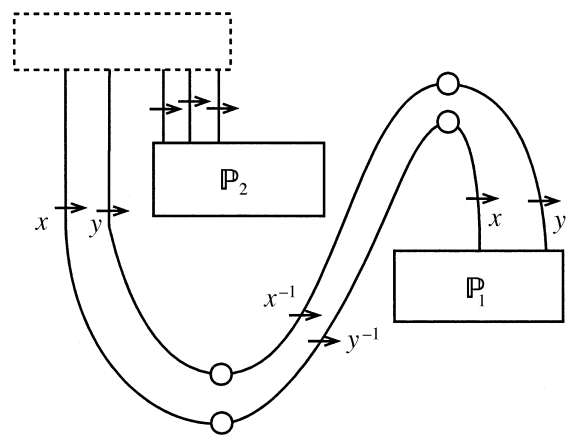

Figure 5

Let $a_{R}=<\mathrm{E}_{R, 1,1}>(R \in \mathbf{r})$.

Proposition. $\Sigma^{*}$ is generated (as a crossed module) by the elements $a_{R}(R \in \mathbf{r})$.

Proof. Let

$$
\mathrm{B}=\mathrm{T}_{1} \mathrm{~A}_{1} \mathrm{~T}_{2} \mathrm{~A}_{2} \cdots \mathrm{T}_{n} \mathrm{~A}_{n} \mathrm{~T}_{n+1}
$$

be a closed path in $\mathcal{D}(\mathcal{P})^{*}$ starting at the reduced word $U$ and ending at the empty word 1. Here the T's are trivial paths and the A's are non-trivial edges. Write $\mathrm{A}_{i}=\left(U_{i}, R_{i}, \varepsilon_{i}, V_{i}\right)(i=1, \cdots, n)$. We claim that

$$
<\mathrm{B}>=\varepsilon_{1}\left[U_{1}\right] \circ a_{R_{1}}+\cdots+\varepsilon_{n}\left[U_{n}\right] \circ a_{R_{n}} .
$$

Let

$$
\mathrm{P}=\mathrm{E}_{1}+\mathrm{E}_{2}+\cdots+\mathrm{E}_{n},
$$

where $\mathrm{E}_{i}=\mathrm{E}_{R_{i}, U_{i}, \varepsilon_{i}}(i=1, \cdots, n)$. Then the right hand side of $(5)$ is $<\mathrm{P}^{*}>$. Now let $\bar{P}$ be the picture obtained from $\mathrm{P}$ by inserting immediately to the right of the $i$ th disc a succession of parallel arcs with total label $V_{i} V_{i}^{-1}(i=1, \cdots, n)$. Then $\bar{P}^{*} \sim \mathrm{P}^{*}$ by (4). Now

$$
\begin{aligned}
\iota(\overline{\mathrm{P}}) & =\iota\left(\mathrm{A}_{1}\right) \tau\left(\mathrm{A}_{1}\right)^{-1} \iota\left(\mathrm{A}_{2}\right) \tau\left(\mathrm{A}_{2}\right)^{-1} \cdots \iota\left(\mathrm{A}_{n}\right) \tau\left(\mathrm{A}_{n}\right)^{-1}, \\
\tau(\overline{\mathrm{P}}) & =\tau\left(\mathrm{A}_{1}\right) \tau\left(\mathrm{A}_{1}\right)^{-1} \tau\left(\mathrm{A}_{2}\right) \tau\left(\mathrm{A}_{2}\right)^{-1} \cdots \tau\left(\mathrm{A}_{n}\right) \tau\left(\mathrm{A}_{n}\right)^{-1},
\end{aligned}
$$

and so we can take $\bar{P}^{*}$ to be $\mathrm{D} \bar{P} \mathrm{D}^{\prime}$ where 


$$
\begin{aligned}
\mathrm{D} & =\mathrm{T}_{1}+\left(\mathrm{T}_{\tau\left(\mathrm{A}_{1}\right)^{-1} \tau\left(\mathrm{A}_{1}\right)}\right)\left(\tau\left(\mathrm{A}_{1}\right)^{-1} \cdot \mathrm{T}_{2}\right)+\cdots+\left(\mathrm{T}_{\tau\left(\mathrm{A}_{n}\right)^{-1} \tau\left(\mathrm{A}_{n}\right)}\right)\left(\tau\left(\mathrm{A}_{n}\right)^{-1} \cdot \mathrm{T}_{n+1}\right) \\
\mathrm{D}^{\prime} & =\mathrm{T}_{\tau\left(\mathrm{A}_{1}\right) \tau\left(\mathrm{A}_{1}\right)^{-1}}^{-1}+\cdots+\mathrm{T}_{\tau\left(\mathrm{A}_{n}\right) \tau\left(\mathrm{A}_{n}\right)^{-1}}^{-1}
\end{aligned}
$$

(see Figure 6). Making use of the defining paths (3) of $\mathcal{D}(\mathcal{P})^{*}$ to eliminate the "bends" we see that $\bar{P}^{*} \sim \mathrm{B}$.
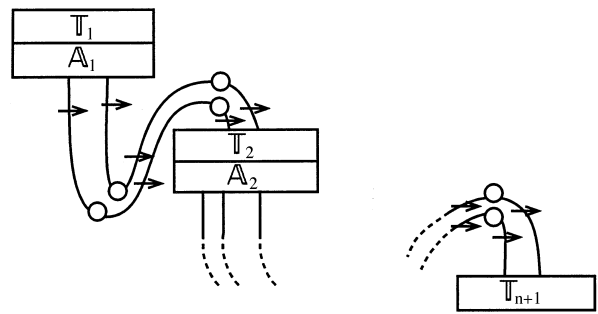

Figure 6

Now since $\Sigma$ is free on the elements $b_{R}=<(R)>(R \in \mathbf{r})$ we have a crossed module homomorphism

$$
\eta: \Sigma \rightarrow \Sigma^{*}, \quad b_{R} \mapsto a_{R}
$$

THEOREM 1. The crossed module homomorphism $\eta$ is an isomorphism.

Proof. We will construct the inverse of $\eta$.

Define a mapping $\psi_{0}$ from the edge set of $\mathcal{D}(\mathcal{P})^{*}$ to $\Sigma$ as follows. Trivial edges are mapped to 0 ; an edge $(U, R, \varepsilon, V)(U, V \in F, R \in \mathbf{r}, \varepsilon= \pm 1)$ is mapped to $<U R^{\varepsilon} U^{-1}>$. Then for any edge A

$$
\partial \psi_{0}(\mathrm{~A})=\left[\iota(\mathrm{A}) \tau(\mathrm{A})^{-1}\right] .
$$

Now $\psi_{0}$ extends to a mapping on paths and it follows from (6) that for any path $\mathrm{P}$

$$
\partial \psi_{0}(\mathrm{P})=\left[\iota(\mathrm{P}) \tau(\mathrm{P})^{-1}\right]
$$

The image of each defining path of $\mathcal{D}(\mathcal{P})^{*}$ is 0 . This is clear for paths of the form (3), and for a path as in (2) we have

$$
\begin{aligned}
\psi_{0}[\mathrm{~A}, \mathrm{~B}]= & \psi_{0}(\mathrm{~A})+[\tau(\mathrm{A})] \cdot \psi_{0}(\mathrm{~B})-\psi_{0}(\mathrm{~A})-[\iota(\mathrm{A})] \cdot \psi_{0}(\mathrm{~B}) \\
= & \partial\left(\psi_{0}(\mathrm{~A})\right) \cdot\left([\tau(\mathrm{A})] \cdot \psi_{0}(\mathrm{~B})\right)-[\iota(\mathrm{A})] \cdot \psi_{0}(\mathrm{~B}) \\
& \quad(\text { using the crossed module structure on } \Sigma) \\
= & 0(\operatorname{using}(6)) .
\end{aligned}
$$

We thus get a well-defined mapping of equivalence classes

$$
<\mathrm{P}>\stackrel{\psi}{\mapsto} \psi_{0}(\mathrm{P})
$$

and in particular, we get a function 


$$
\psi: \Sigma^{*} \rightarrow \Sigma
$$

Now $\psi$ is a group homomorphism, since for any $<\mathrm{P}_{1}>,<\mathrm{P}_{2}>\in \Sigma^{*}$ we have

$$
\begin{aligned}
\psi\left(<\mathrm{P}_{1}>+<\mathrm{P}_{2}>\right) & =\psi_{0}\left(\left(\left(\mathrm{P}_{1} \cdot \iota\left(\mathrm{P}_{2}\right)\right) \mathrm{P}_{2}\right)^{*}\right) \\
& =\psi_{0}\left(\left(\mathrm{P}_{1} \cdot \iota\left(\mathrm{P}_{2}\right)\right) \mathrm{P}_{2}\right) \\
& =\psi_{0}\left(\mathrm{P}_{1} \cdot \iota\left(\mathrm{P}_{2}\right)\right)+\psi_{0}\left(\mathrm{P}_{2}\right) \\
& =\psi_{0}\left(\mathrm{P}_{1}\right)+\psi_{0}\left(\mathrm{P}_{2}\right) \\
& =\psi<\mathrm{P}_{1}>+\psi<\mathrm{P}_{2}>.
\end{aligned}
$$

Also, it is easily checked that $\psi$ respects the $\hat{F}$-action, and it follows from (7) that $\partial \psi=\partial^{*}$. Hence $\psi$ is a crossed module homomorphism.

Since $\psi \eta$ agrees with the identity on the generating set $a_{R}(R \in \mathbf{r})$ of $\Sigma^{*}, \psi \eta=1$. Similarly $\eta \psi=1$.

This proves the theorem.

Note that, by restriction, we get a mutually inverse pair of isomorphisms

$$
\pi_{2}(\hat{\mathcal{P}})=\operatorname{Ker} \partial \underset{\psi}{\stackrel{\eta}{\rightleftarrows}} \operatorname{ker} \partial^{*}=\pi_{1}\left(\mathcal{D}(\mathcal{P})^{*}, 1\right)
$$

The $G$-action on $\pi_{2}(\hat{\mathcal{P}})$ induces a $G$-action on $\pi_{1}\left(\mathcal{D}(\mathcal{P})^{*}, 1\right)$ by the rule

$$
\bar{W}_{\circ}<\mathrm{P}>=<\left(W \cdot \mathrm{P} \cdot W^{-1}\right)^{*}>\left(\bar{W} \in G,<\mathrm{P}>\in \pi_{1}\left(\mathcal{D}(\mathcal{P})^{*}, 1\right)\right),
$$

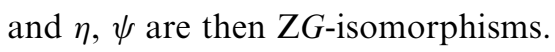

3. The fundamental groups of $\mathcal{D}(\mathcal{P})^{*}$. Let $U \in F$. We have a well-defined group homomorphism

$$
\begin{gathered}
\phi_{U}: \pi_{1}\left(\mathcal{D}(\mathcal{P})^{*}, 1\right) \rightarrow \pi_{1}\left(\mathcal{D}(\mathcal{P})^{*}, U\right), \\
<\mathrm{B}>\mapsto<\mathrm{B} \cdot U>
\end{gathered}
$$

This is in fact an isomorphism, for consider the (well-defined) function

$$
\begin{gathered}
\theta_{U}: \pi_{1}\left(\mathcal{D}(\mathcal{P})^{*}, U\right) \rightarrow \pi_{1}\left(\mathcal{D}(\mathcal{P})^{*}, 1\right) \\
<\mathrm{P}>\mapsto<\left(\mathrm{P} \cdot U^{-1}\right)^{*}>
\end{gathered}
$$

Now $\theta_{U} \phi_{U}=1$, for if $\mathrm{B}$ is a spherical monoid picture with $\iota(\mathrm{B})=1$ then $\left(\mathrm{B} \cdot U U^{-1}\right)^{*} \sim$ $\mathrm{B}^{*}=\mathrm{B}$ by (4). Also, $\phi_{U} \theta_{U}=1$, for if $\mathrm{P}$ is a spherical monoid picture with $\iota(\mathrm{P})=U$ then (see Figure 7)

$$
\left(\mathrm{P} \cdot U^{-1}\right)^{*} \cdot U \sim \mathrm{P} .
$$

Thus $\theta_{U}, \phi_{U}$ are mutually inverse isomorphisms. 

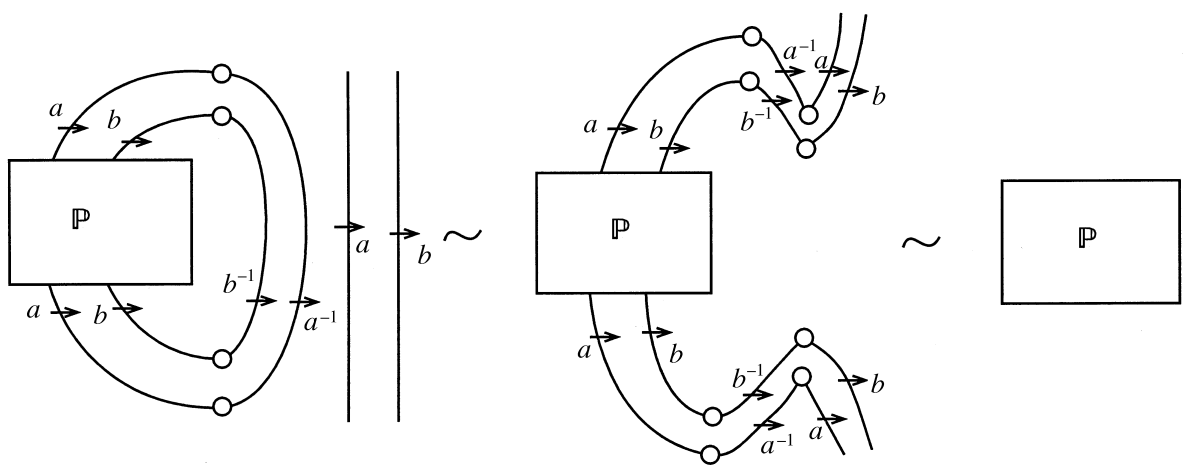

Figure 7

We will need the following result.

Lemma. Let $\mathrm{P}$ be a spherical monoid picture over $\mathcal{P}$ with $\iota(\mathrm{P})=U$. Suppose $W$, $V \in F$ are such that $W U V={ }_{G} 1$. Let $\mathrm{D}$ be any path in $\mathcal{D}(\mathcal{P})^{*}$ from 1 to $W U V$. Then in $\pi_{1}\left(\mathcal{D}(\mathcal{P})^{*}, 1\right)$ we have

$$
<\mathrm{D}(W \cdot \mathrm{P} \cdot V) \mathrm{D}^{-1}>=\bar{W} \circ \theta_{U}<\mathrm{P}>.
$$

This can be seen geometrically as follows. First note that $V^{-1} U^{-1} W^{-1}={ }_{\mathrm{G}} 1$ so there is a path $\bar{D}$ in $\mathcal{D}\left(\mathcal{P}^{*}\right)$ from 1 to $V^{-1} U^{-1} W^{-1}$. Then we have the equivalence as in Figure 8 (where for simplicity we have taken $W, U, V$ to each consist of a single letter).
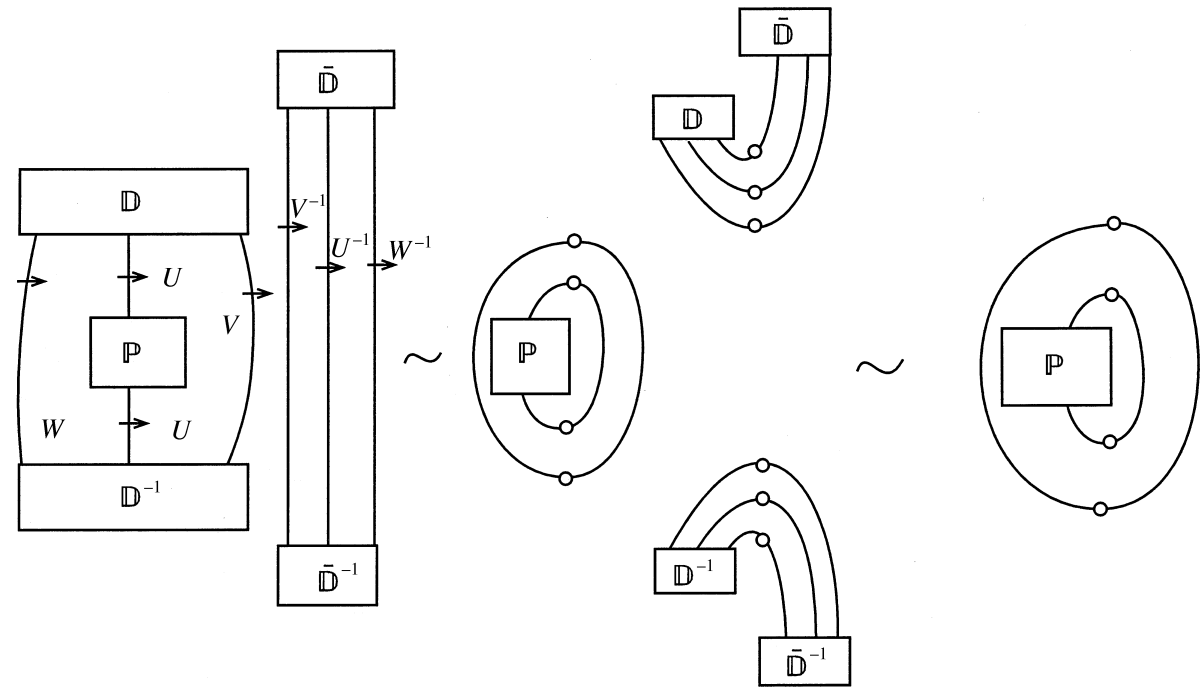

Figure 8

Theorem 2. $\mathcal{P}$ is of finite derivation type if and only if the left ZG-module $\pi_{1}\left(\mathcal{D}(\mathcal{P})^{*}, 1\right)$ is finitely generated.

Proof. First suppose that $\mathcal{P}$ has finite derivation type. Then there is a finite collection $X$ of spherical monoid pictures over $\mathcal{P}$ such that the 2-complex $\mathcal{D}(\mathcal{P})^{X}$ obtained from $\mathcal{D}(\mathcal{P})$ by adjoining the defining paths 


$$
W \cdot \mathrm{P} \cdot V \quad(W, V \in F, \mathrm{P} \in X)
$$

has trivial fundamental groups.

Let $\mathrm{B}$ be any spherical monoid picture with $\iota(\mathrm{B})=1$. Then $\mathrm{B}$ is homotopic in $\mathcal{D}(\mathcal{P})$ (and hence in $\mathcal{D}(\mathcal{P})^{*}$ ) to a product of the form

$$
\prod_{i=1}^{n} \mathrm{D}_{i}\left(W_{i} \cdot \mathrm{P}_{i} \cdot V_{i}\right)^{\varepsilon_{i}} D_{i}^{-1}
$$

where $\mathrm{P}_{i} \in X, \quad \varepsilon_{i}= \pm 1, \quad W_{i}, \quad V_{i} \in F, \quad \mathrm{D}_{i}$ is some path in $\mathcal{D}(\mathcal{P})$ with $\iota\left(\mathrm{D}_{i}\right)=1$, $\tau\left(D_{i}\right)=\iota\left(W_{i} \cdot P_{i} \cdot V_{i}\right)(i=1, \cdots, n)$. Hence in $\pi_{1}\left(\mathcal{D}(\mathcal{P})^{*}, 1\right)$ we have

$$
\begin{aligned}
<B> & =\sum_{i=1}^{n} \varepsilon_{i}<D_{i}\left(W_{i} \cdot P_{i} \cdot V_{i}\right) D_{i}^{-1}> \\
& =\sum_{i=1}^{n} \varepsilon_{i} \bar{W}_{i} \circ \theta_{l\left(P_{i}\right)}<P_{i}>\text { (by the Lemma). }
\end{aligned}
$$

Thus the module $\pi_{1}\left(\mathcal{D}(\mathcal{P})^{*}, 1\right)$ is generated by the elements

$$
\left\{\theta_{l(\mathrm{P})}<\mathbf{P}>: \mathbf{P} \in X\right\}
$$

Conversely, suppose there is a finite set $Y$ of spherical monoid pictures (each starting at 1) such that the elements $<\mathrm{B}>(\mathrm{B} \in Y)$ generate $\pi_{1}\left(\mathcal{D}(\mathcal{P})^{*}, 1\right)$ as a module. Let $\mathrm{P}$ be any spherical monoid picture, and suppose that $\iota(\mathrm{P})=U$. Then

$$
\theta_{U}<P>=\sum_{i=1}^{n} \varepsilon_{i} \bar{W}_{i} \circ<\mathrm{B}_{i}>
$$

where $\mathrm{B}_{\mathrm{i}} \in Y, W_{i} \in F, \varepsilon_{i}= \pm 1(i=1, \cdots, n)$. Thus in $\pi_{1}\left(\mathcal{D}(\mathcal{P})^{*}, U\right)$ we have

$$
\begin{aligned}
<\mathrm{P}> & =\prod_{i=1}^{n} \phi_{U}\left(\bar{W}_{i} \circ<\mathrm{B}_{i}>\right)^{\varepsilon_{i}} \\
& =\prod_{i=1}^{n}<\left(\mathrm{T}_{W_{i} W_{i}^{-1}} \cdot U\right)\left(W_{i} \cdot \mathrm{B}_{i}^{\varepsilon_{i}} \cdot W_{i}^{-1} U\right)\left(\mathrm{T}_{W_{i} W_{i}^{-1}} \cdot U\right)^{-1}>.
\end{aligned}
$$

Consequently, we see that if we adjoin to $\mathcal{D}(\mathcal{P})^{*}$ the additional defining paths

$$
W \cdot \mathrm{B} \cdot V \quad(W, V \in F, \mathrm{~B} \in Y)
$$

then all fundamental groups of the resulting complex are trivial. Thus if $X$ consists of the pictures in $Y$ together with the pictures of the form (3), then $\mathcal{D}(\mathcal{P})^{X}$ has trivial fundamental groups, and so $\mathcal{P}$ is of finite derivation type.

Acknowledgement. I thank Victor Guba for useful discussions relating to this paper. 


\section{REFERENCES}

1. W. A. Bogley and S. J. Pride, Calculating generators of $\pi_{2}$, in: Low Dimensional Homotopy Theory and Combinatorial Group Theory, C. Hog-Angeloni, W. Metzler, A. Sieradski (eds.) (Cambridge University Press, 1993), 157-188.

2. K. S. Brown, Cohomology of groups (Springer-Verlag, 1982).

3. R. Brown and J. Huebschmann, Identities among relations, in: Low-Dimensional Topology, R. Brown and T. L. Thickstun (eds.), London Mathematical Society Lecture Notes Series 48 (1982), 153-202.

4. R. Cremanns and F. Otto, Finite derivation type implies the homological finiteness condition $\mathrm{FP}_{3}$, Journal of Symbolic Computation 18 (1994), 91-112.

5. R. Cremanns and F. Otto, For groups the property of having finite derivation type is equivalent to the homological finiteness condition $\mathrm{FP}_{3}$, Journal of Symbolic Computation 22 (1996), 155-177.

6. M. N. Dyer, Crossed modules and $\pi_{2}$ homotopy modules, in: Low-Dimensional Homotopy and Combinatorial Group Theory, C. Hog-Angeloni, W. Metzler, A. Sieradski (eds.) (Cambridge University Press, 1993), 125-156.

7. V. Guba and M. Sapir, Diagram groups, Memoirs of the American Mathematical Society, No. 620 (A.M.S., 1997).

8. V. Kilibarda, On the algebra of semigroup diagrams, Internat. J. Algebra Comput. 7 (1997), 313-338.

9. Y. Lafont, A new finiteness condition for monoids presented by complete rewriting systems (after Craig C. Squier). J. Pure Appl. Algebra 98 (1995), 229-244.

10. S. J. Pride, Identities among relations of group presentations, in: Group theory from a Geometrical Viewpoint, E. Ghys, A. Haefliger, A. Verjovsky (eds.), (World Scientific, Singapore, New Jersey, London, Hong Kong, 1991), 687-717.

11. S. J. Pride, Geometric methods in combinatorial semigroup theory, in: Semigroups, Formal Languages and Groups, J. Fountain (ed.) (Kluwer Publishers, 1995), 215-232.

12. S. J. Pride, Low-dimensional homotopy theory for monoids, Internat. J. Algebra Comput. 5 (1995), 631-649.

13. C. C. Squier, A finiteness condition for rewriting systems, revision by F. Otto and Y. Kobayashi, Theoretical Computer Science 131 (1994), 271-294.

14. J. H. C. Whitehead, Combinatorial homotopy II. Bull. Amer. Math. Soc. 55 (1949), 453-496. 